\title{
Reduce of bearing strength of the bent reinforce-concrete elements on a sloping section with the corrosive damage of transversal armature
}

\author{
Ashot Tamrazyan ${ }^{1}$, and Dmitriy Popov ${ }^{1 *}$ \\ ${ }^{1}$ Moscow State University of Civil Engineering, Moscow,Russia
}

\begin{abstract}
: the technique of calculation of the reinforced concrete element exposed to the aggressive environment is considered, the quantitative reduction estimation of power resistance of concrete and armature is given.
\end{abstract}

\section{Introduction}

Corrosive influences entail considerable degradation of bearing strength of reinforceconcrete elements of building and constructions in the period of their exploitation. [1]. The protracted exploitation without realization of events on major repairs leads to a problem about structural safety and vitality of building. Tasks on reduction of bearing strength and as a result prognostication of risks of bringing down of building, which is inalienable part of modern science [2-4].

Numerous authors engaged in the account of decline of power resistance, construction of models of empiric dependences and depth-finding of corrosive damage of reinforceconcrete element [5-7].

The probability of breaking a bent element along an inclined section is equally probable, as in the normal section, so it becomes necessary to analyze this case

As is generally known, there are three types of calculation and accordingly destruction of the bent reinforce-concrete element on sloping sections: fracture from the action of flexion moment $\mathrm{M}$; breaking up of the compressed concrete on a stripe between sloping cracks; change on a sloping section from the action of transversal force of $Q$.

The strength of the element along the inclined cross section to the action of shear force under the influence of an aggressive environment cannot be determined by means of normative literature within the framework of the actual evaluation of the bearing capacity of the element.

This complexity is stipulated by variation of types of damage (pitting, continuous, uneven), kinetics of advancement (colmatation, filtration, avalanche), presence and thicknesses of zones of corrosive damage (fig 1). Under the influence of the aggressor, the physical and mechanical characteristics of the reinforcement and concrete change.

\footnotetext{
* Corresponding author: popovds89@mail.ru
} 
This leads to the need of solving new problems with variable cross-section of transverse reinforcement, heterogeneous strength of concrete, the length of the projection of the inclined section to determine the actual bearing capacity of the element at different stages of operation.

\section{Methods}

In this article the sloping section of reinforce-concrete element is examined at the one-sided contact of aggressive environment with the external fibre of the compressed zone. It is used two and three-zone layout of power resistance chart [8] (fig. 1). Reduction of crosssectional reinforcement cross-section is achieved by entering a correction factor $\omega_{s w}$. On an image 1 reduction of area of transversal armature is shown in a section depending on character of corrosive destruction. So at existence of the destroyed fibre layer $\left(z^{*}>0\right)$, power resistance of transversal armature and concrete on a height $z^{*}$ is not taken into account in calculations (fig .1B)

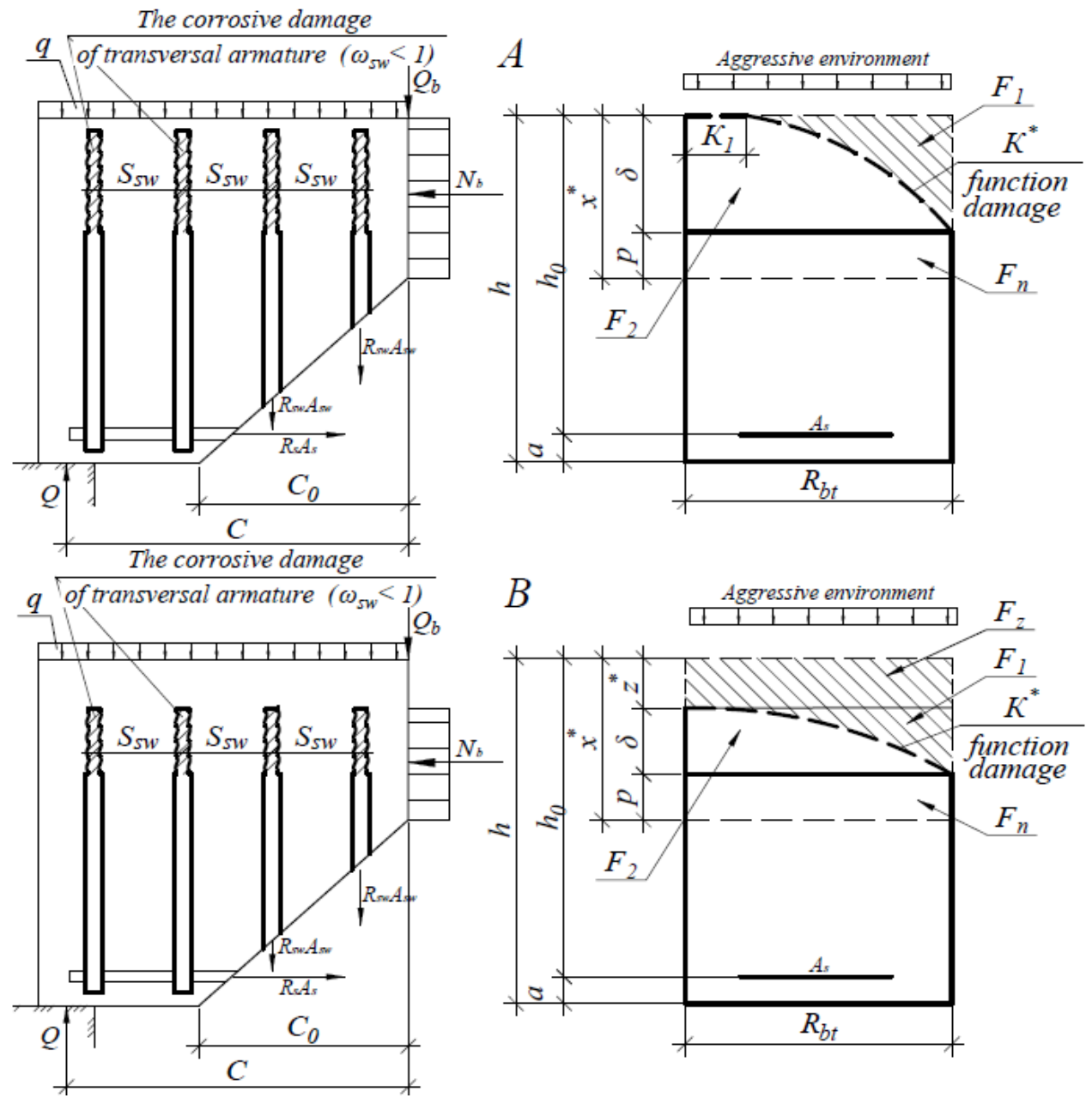

Fig. 1.Calculation charts of the bent reinforce-concrete element damaged by corrosion : A - in default of the fully lost resistance of fibre layer $\left(z^{*}=0\right)$; B $\quad$ - at presence of the destroyed fibre layer $\left(z^{*}>0\right)$ 
Here $z^{*}$ - is a thickness of complete corrosive destruction of concrete; $\delta$ - it is a thickness of incomplete corrosive damage of concrete; $\mathrm{p}$ is a thickness of uncrippled concrete; $a, x^{*}, h, h_{0}$ - are geometrical sizes of concrete body (standard); $K^{*}$ - is a curve of function of damages (coefficient of maintenance of initial mechanical descriptions); $K_{l}$ - is the fixed value of stake of maintenance of durability of concrete on an external fibre; $\mathrm{Fz}^{*}$ is a size of the lost part of power resistance of concrete; $F_{1}$ - is a size of the lost part of power resistance of concrete; $F_{2}$ - is a size of the saved part of power resistance of concrete; $F S$ - is a size of power resistance of longitudinal armature; $S_{s w}$ - is a step of transversal armature; $\omega_{s w}$ - is a coefficient of damage of transversal armature; $C$ - is distance from the top of calculation sloping section to support; $C_{0}$ - is a projection of sloping calculation section; $N_{b}$ - resultant normal tensions in a concrete.

Classification of a large number of corrosive processes of concrete is combined into three main types. In the first form of corrosion, the composition of concrete $\mathrm{Ca}(\mathrm{OH})_{2}$ dissolves under the influence of atmospheric precipitation, the second type is characterized by the interaction with acids contained in water and the third type of corrosion of concrete occurs as a result of crystallization of hardly soluble substances. Under natural conditions, corrosion of one or another species is rarely found apart from the others, but usually one species predominates. The author does not consider any kind of corrosion damage, taking into account the reduction in the mechanical characteristics of materials exposed to the corrosive environment

Researches of the bent corrosive damaged reinforce-concrete elements on a sloping section in home literature on the real stage small enough $[9,10]$.

In foreign literature it is possible to distinguish some works $[11,12]$ which give the results of researches of durability and character of destructions of the reinforce-concrete beams on a sloping section, exposed to influence of aggressive environment.

Durability of sloping section from сдвиговых destructions (caused by transversal force) without the "unbended" re-enforcement it is obliged to be provided concordantly [13]. In this work [10] the calculation of sloping section is executed taking into account the corrosive damages of reinforce-concrete element.

$$
Q^{*}=Q_{s w}^{*}+Q_{b}^{*}
$$

where, $Q^{*}$ - remaining resource of power resistance of the damaged reinforce-concrete element on a vertical cut; $Q_{s w}^{*}$ - is a stake of this resource, being transversal armature; $Q_{b}{ }^{*}$ stake of this resource, being on the cut away part of the damaged concrete.

Pursuant to [9], it is possible to write down :

$$
\begin{aligned}
& Q_{s w}^{*}=\sum \omega_{s w} A_{s w} R_{s w}=q_{s w}^{*} c \\
& q_{s w}^{*}=\frac{\omega_{s w} A_{s w} R_{s w}}{S_{s w}} ; Q_{s w}^{*}=\omega_{s w} A_{s w} R_{s w} \frac{c}{S_{s w}} \\
& Q_{b}^{*}=\frac{M^{*}}{c} ; M^{*}=\varphi\left(h_{0}-z^{*}\right) N_{b}^{*} \\
& N_{b}^{*}=b\left[\left(h_{0}-z^{*}\right) R_{b t}-F_{1}\right] F_{1}=\frac{1+k_{1}}{3} \delta b R_{b t}
\end{aligned}
$$

where, $S_{s w}$ - is distance between transversal reinforcing bars; $c$ - is an ichnography of sloping crack; $b$ - is a width of element; $\varphi=1.5$ integral empiric correcting coefficient; $R_{b t}$ - ultimate strength of concrete by tension; $q_{s w}$ - is effort in a transversal armature by length of element unit; $\omega$ sw is a coefficient of damage of transversal armature; $A_{s w}$ - is an area of 
section of transversal armature; $R_{s w}$ - a calculated resistance of transversal armature; $F_{l}$ - is a size of the lost part of power resistance if $z^{*}=0$ and $K 1>0$.

Now the sought after resistance of the damaged section to transversal force will get a record:

$$
Q^{*}=q_{s w}^{*} c+\frac{M^{*}}{c}
$$

for that a value of $\mathrm{C}$ is from the condition of minimization (4) at $c$ :

$$
\frac{d Q^{*}}{d c}=0, \text { т.е. } c=\sqrt{\frac{M^{*}}{q_{s w}^{*}}}
$$

Developing a method [10] for next corrosive damages we will get:

- on condition of damage: $z^{*}>0, K_{I}=0,0<K^{*}<1$. i.e. at existence of the fully destroyed fibre layer of $z^{*}>0$ and absence of the saved durability on the fibre layer of $K_{l}=0$, in equalization (6) from the working height of section subtracted the fully destroyed layer of $z^{*}$ and $1 / 3$ part of the not fully destroyed (transitional) layer, and from the shoulder of force the size of the fully destroyed layer of $z *$ is subtracted.

$$
M^{*}=1,5 b h_{0}^{2} R_{b t}=1,5 b\left(h_{0}-z^{*}\right) R_{b t}\left[h_{0}-\left(z^{*}+\frac{1}{3} \delta\right)\right]
$$

- on condition of damage: $z^{*}=0, K_{l}=0,0<K^{*}<1$. i.e. when the fully destroyed fibre layer of $z^{*}=0$ and saved durability is absent on the fibre layer of $K_{I}=0$ in equalization (7) from the working height of section subtracted only $1 / 3$ part of the not fully destroyed (transitional) layer.

$$
M^{*}=1,5 b h_{0}^{2} R_{b t}=1,5 b h_{0} R_{b t}\left(h_{0}-\frac{1}{3} \delta\right)
$$

Losses of power resistance of transversal effort from influence of aggressive environment defining in a percentage ratio is possible on a formula:

$$
P=\frac{Q-Q^{*}}{Q} 100
$$

where, $Q=Q_{s w}+Q_{b}$ is primary (project) power resistance of element on a vertical cut; $P$ - are losses of power resistance in percents.

Being base on an offer methodology (4), (8) the calculation of reinforce-concrete beam is executed on a sloping section at two different terms of damages.

Condition of damage 1: $z^{*}=0, \omega_{s w}=1$ and $0<\delta_{\max }<x$, id est, when an area of complete destruction is not, a transversal armature is not damaged, and the area of incomplete corrosive damage of concrete is varied from 0 to $x$.

Condition of damage 2: $z^{*}=0, \omega_{s w}=0,5$ and $0<\delta_{\max }<x$, id est, when an area of complete destruction is not, the area of cross-sectional is diminished on $50 \%$, and the area of incomplete corrosive damage of concrete is varied from 0 to $x$. 


\section{Results}

We will accept some conditional descriptions of durability and corrosive damages : the bent element of rectangular section of $h=600 \mathrm{~mm}, b=300 \mathrm{~mm}, a=30 \mathrm{~mm}$, concrete of B15 $\left(R_{b}=8,5 \mathrm{MPa}, R_{b t}=0,75 \mathrm{MPa}\right)$, with re-enforcement in the stretched zone by an armature by the class of A400 $(R s=350 \mathrm{MPa})$, border relative height of the compressed zone $\xi_{\mathrm{R}}=0,533$, transversal armature of class of A240 $\left(R_{s w}=170 \mathrm{MPa}\right), A_{s w}=157 \mathrm{~mm}^{2}$, with the step of $S_{s w}=100 \mathrm{~mm}$. The corrosive damage of longitudinal working armature is $\omega_{s}=1$. The maximal depth of damages $\delta_{\max }$ is accepted to the equal border height of the compressed zone of section $\delta_{\max }=x$, where $x=\xi_{R} \cdot h_{0}=30,38 \mathrm{~cm}$.

The results of calculation are presented on the fig. 2, that illustrates the relation of depth of damage and to the decline of bearing strength of element in percents, so at the first set condition the decline of power resistance arrives at no more than $10 \%$, but if to accept the terms of development of corrosion in a transversal armature, on the example of $50 \%$ reduction of area of cross-sectional, general losses develop to $36 \%$.

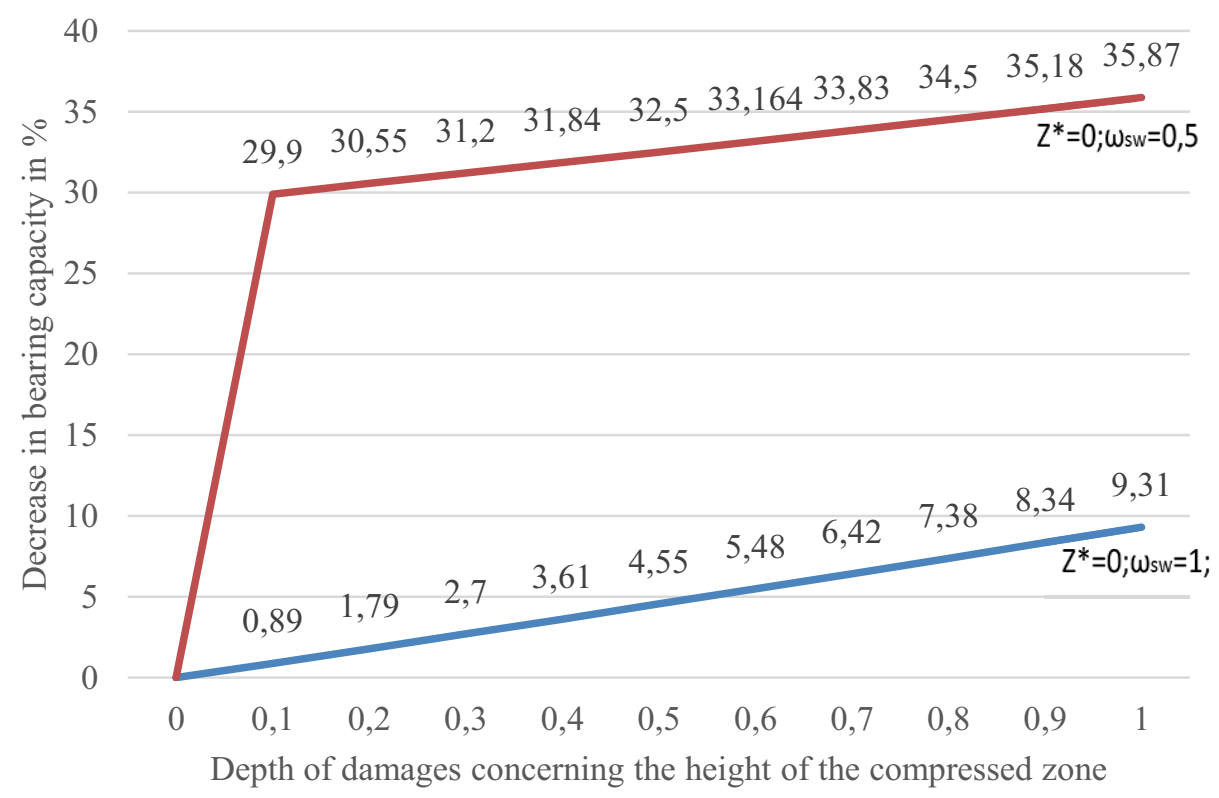

Fig.2 Chart of loss of power resistance of reinforce-concrete element on a sloping section at the increase of depth of corrosive damage $\delta$.

As is generally known bearing strength of reinforce-concrete element is provided by a concrete and armature, coming from principle of calculation on [13] the general losses of power resistance of element on a sloping section from influence of aggressive environment will consist of $57 \%$ due to a concrete and $43 \%$ armature.

According to [13], the strength condition for the oblique section must be observed:

$$
Q<Q^{*}
$$

In the event that condition (9) is not observed, the element must be strengthened. One of the possible methods of strengthening is the installation of additional vertical clamps, the necessary cross-sectional area in the corresponding zone. 


$$
Q<Q_{b}^{*}+Q_{s w}^{*}+Q_{f w}
$$

Where, $Q_{f w}$ - is the transverse force perceived by the additionally mounted vertical clamps.

$$
Q_{f w}=\frac{A_{f w} R_{f w} c}{S_{f}}
$$

Where, $S_{f}$ - is the distance between the additional transverse clamps; C is the horizontal projection of the inclined crack; $A_{f w}$ - cross-sectional area of additional transverse clamps; $R_{f w}$ - is the design resistance of the additional clamps.

\section{Conclusion}

Thus, the method of calculation is considered on the sloping section of the reinforceconcrete corrosive damaged element. It is set that the decline of power resistance of the corrosive damaged reinforce-concrete element arrives at to $36 \%$ at reduction of area of transversal armature on $50 \%$. The decline of bearing strength on $57 \%$ takes place due to a concrete, and $43 \%$ due to an armature.

\section{References}

1. E. Larionova MSSU Announcer.6, 26 (2016).

2. A.Tamarzyan.M.Orlova Construction in housing. 6, 32 (2015).

3. A.Tamarzyan.M.Orlova MSSU Announcer. 4, 181 (2014)

4. A.Tamarzyan,V. Falikman Construction and reconstruction. 3(65), 71 (2016)

5. A. Popesku Capacity of reinforce-concrete constructions subject to corrosion. SASU, 182 (1996).

6. G. Smolyago, A.Krychkov, S. Drokin, A. Dronov. Announcer of Belgorod State technological university the name of. Shukhov. 2, 22, (2014).

7. O. Morozova, S, Markov, I. Stavskaya Construction and reconstruction. 3(65), 71 (2016)

8. V Bondarenko Concrete and reinforced concrete. 2, 25 (2008).

9. N. Klyueva, D. Karpenko, A. Kashavtsev Structural mechanics of engineering constructions and buildings. 5, 77 (2015)

10. B. Yagupov., R. Migal Concrete and reinforced concrete. 3, 28 (2007)

11. I . Khan., R. Francois,. A. Castel. Materials and Structures. V 47, I 9, 1467 (2014)

12. B.Almassri, J. Barros, F. Mahmoud, R. Francois. Composite Structures. V 131, 731 (2015)

13. СП 63.13330.2012. Constructions from concrete and reinforced concrete. 